\title{
Editorial
}

\section{Theoretical and Numerical Results for Fractional Difference and Differential Equations}

\author{
Qasem M. Al-Mdallal, ${ }^{1}$ Thabet Abdeljawad, ${ }^{2}$ and Mohamed A. Hajji ${ }^{1}$ \\ ${ }^{1}$ Department of Mathematical Sciences, United Arab Emirates University, P.O. Box 17551, Al Ain, Abu Dhabi, UAE \\ ${ }^{2}$ Department of Mathematics and Physical Sciences, Prince Sultan University, P.O. Box 66833, Riyadh 11586, Saudi Arabia \\ Correspondence should be addressed to Qasem M. Al-Mdallal; q.almdallal@uaeu.ac.ae
}

Received 19 July 2017; Accepted 19 July 2017; Published 20 August 2017

Copyright (C) 2017 Qasem M. Al-Mdallal et al. This is an open access article distributed under the Creative Commons Attribution License, which permits unrestricted use, distribution, and reproduction in any medium, provided the original work is properly cited.

The fractional calculus is relatively an old topic dating back to more than 300 years. In 1695, L'Hopital wrote to Leibniz asking him about the notation of the $n$th derivative $\left(D^{n} f / d x^{n}\right)$ which was mentioned for the first time in his publications. L'Hopital asked Leibniz about the result if $n=$ 1/2. Leibniz's response was as follows: An apparent paradox, from which one day useful consequences will be drawn. This communication was what created the so-called fractional calculus. However, the gate to the fractional calculus subject was opened in 1832 by Liouville in a series of papers from 1832 to 1837. Later, the intensive investigations on the results of Liouville by Riemann led to the construction of the RiemannLiouville fractional integral operator, given by

$$
J^{\alpha} y(x)=\frac{1}{\Gamma(\alpha)} \int_{a}^{x}(x-t)^{\alpha-1} y(t) d t
$$

where $y \in L_{1}(a, b)$ and $\alpha \in \mathbb{R}^{+}$. Notice that this definition for fractional integral operator had been a valuable cornerstone in fractional calculus subject and had led to the left RiemannLiouville fractional derivative, defined by

$$
\begin{aligned}
D_{R}^{(\alpha)} y(x) & =\frac{d^{n}}{d x^{n}} J^{n-\alpha} y(x) \\
& =\frac{1}{\Gamma(n-\alpha)} \frac{d^{n}}{d x^{n}} \int_{a}^{x}(x-t)^{n-\alpha-1} y(t) d t
\end{aligned}
$$

where $y \in C^{n}[a, b]$ and $n=[\alpha]$ is the smallest integer greater than or equal to $\alpha$.

Since then, many mathematicians were involved in the development of fractional calculus subject up to the middle of the twentieth century such as P. S. Laplace, J. B. J. Fourier, N. H. Abel, H. Holmgren, A. K. Grunwald, A. V. Letnikov, H. Laurent, P. A. Nekrassov, A. Krug, I. Hadamard, O. Heaviside, S. Pincherle, G. H. Hardy and I. E. Littlewood, H. Weyl, P. Levy, A. Marchaud, H. T. Davis, E. L. Post, A. Zygmund, E. R. Love, A. Erdelyi, H. Kober, D. V. Widder, and M. Riesz and W. Feller; for intensive historical background the reader is referred to the novel work on fractional calculus by Ross [1].

An alternative and novel definition of fractional derivative was introduced by Caputo in 1967, given by

$$
\begin{aligned}
D_{C}^{(\alpha)} y(x) & =J^{n-\alpha} \frac{d^{n}}{d x^{n}} y(x) \\
& =\frac{1}{\Gamma(n-\alpha)} \int_{a}^{x}(x-t)^{n-\alpha-1} y^{(n)}(t) d t
\end{aligned}
$$

where $y \in C^{n}[a, b]$ and $n=[\alpha]$.

Notice that this definition produces different properties than Riemann-Liouville fractional derivative. It should be pointed out that although the literature reveals several types of fractional derivative (such as Grunwald-Letnikov derivative, Sonin-Letnikov derivative, Hadamard derivative, Marchaud derivative, Riesz derivative, Riesz-Miller derivative, Miller-Ross derivative, Weyl derivative, and Erdelyi-Kober derivative), still the Riemann-Liouville and Caputo fractional derivatives are the most commonly used ones.

It is well-known that there is still a lack of geometric and physical interpretation of fractional integration and differentiation. However, the fractional derivatives have been 
proved to be a powerful technique for solving integral and differential equations resulting from several physical models. For instance, linear viscoelasticity is the field of the most applications of fractional calculus since it models hereditary phenomena with long memory. In fact, we may refer to other applications like finance, stochastic processes, signal processing, automatic control, electromagnetic theory, elasticity, diffusion and advection phenomena, and so on as applications of fractional calculus (the reader is referred to the survey paper [2]).

In this special issue, we focus on the recent theoretical and numerical studies on fractional ordinary differential equations, fractional partial differential equations, fractional stochastic differential equations, fractional delay differential equations, fractional difference equations, and fractional difference dynamical systems. We hope that the papers of this special issue will enrich our readers and stimulate researchers to extend, generalize, and apply the established results.

\section{Qasem M. Al-Mdallal Thabet Abdeljawad Mohamed A. Hajji}

\section{References}

[1] B. Ross, "A brief history and exposition of the fundamental theory of fractional calculus," in Fractional Calculus and Its Applications, vol. 457 of Lecture Notes in Mathematics, pp. 1-36, Springer Berlin Heidelberg, Berlin, Germany, 1975.

[2] J. T. Machado, V. Kiryakova, and F. Mainardi, "Recent history of fractional calculus," Communications in Nonlinear Science and Numerical Simulation, vol. 16, no. 3, pp. 1140-1153, 2011. 


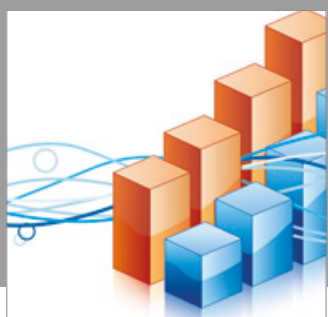

Advances in

Operations Research

vatersals

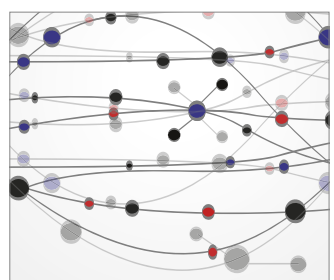

\section{The Scientific} World Journal
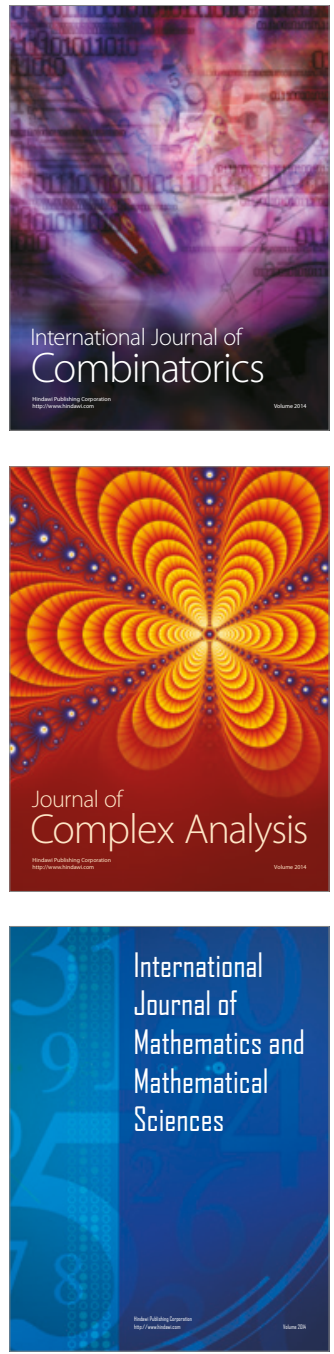
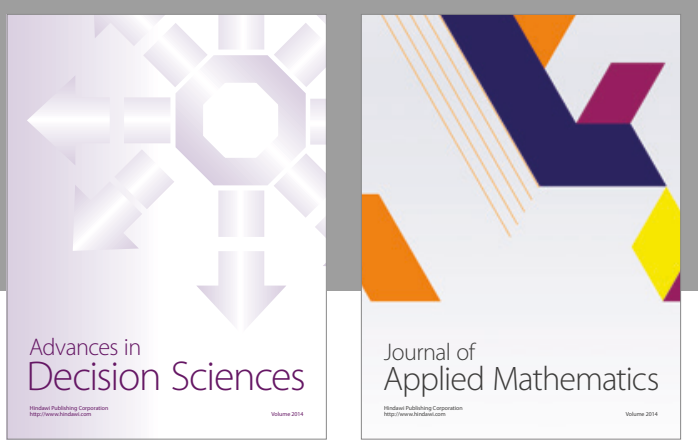

Algebra

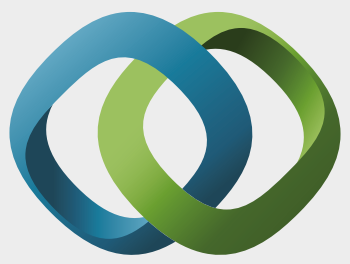

\section{Hindawi}

Submit your manuscripts at

https://www.hindawi.com
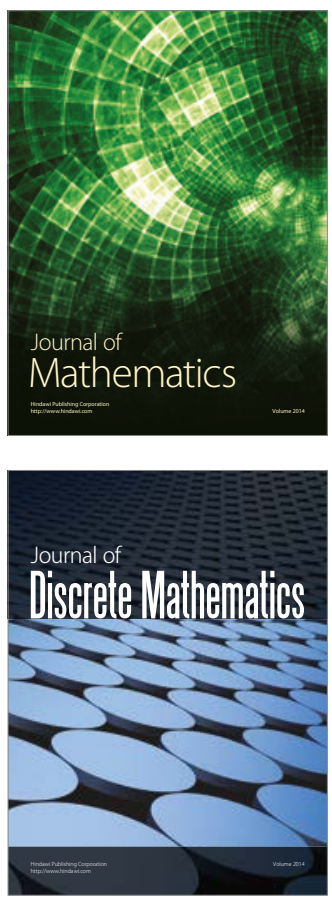

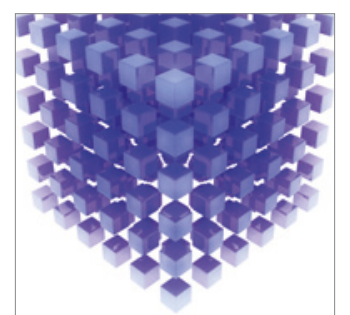

Mathematical Problems in Engineering
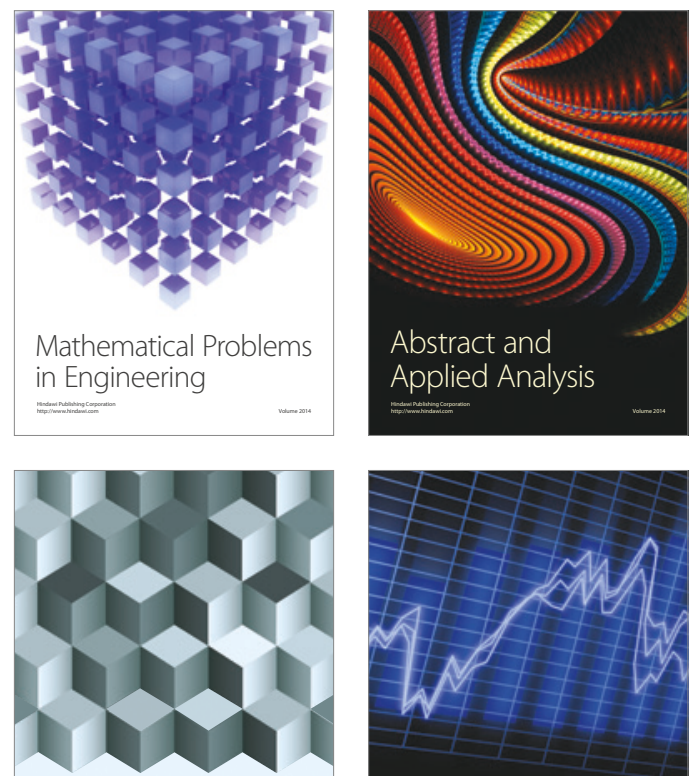

Journal of

Function Spaces

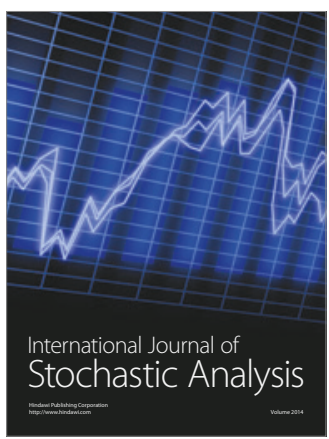

Probability and Statistics
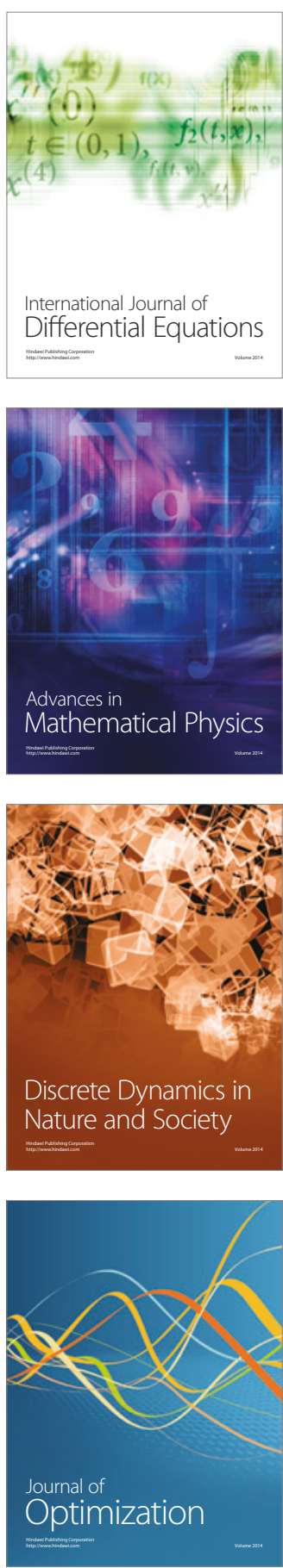